\title{
0-3
}

\section{ICRF specific plasma wall interactions in JET with the ITER-like wall}

\author{
V1. Bobkov ${ }^{\mathrm{a}, *}$ G.Arnoux ${ }^{\mathrm{e}}$ S.Brezinsek ${ }^{\mathrm{b}}$ J.W.Coenen ${ }^{\mathrm{b}}$ L.Colas ${ }^{\mathrm{c}}$ \\ M.Clever ${ }^{\mathrm{b}}$ A.Czarnecka ${ }^{\mathrm{d}}$ F.Braun $^{\mathrm{a}}{ }^{\text {R.Dux }}{ }^{\mathrm{a}}$ A.Huber ${ }^{\mathrm{b}}$ P.Jacquet $^{\mathrm{e}}$ \\ C.Klepper ${ }^{\mathrm{c}}$ E.Lerche ${ }^{\mathrm{f}}$ C.Maggi ${ }^{\mathrm{a}}$ F.Marcotte ${ }^{\mathrm{c}}$ M.Maslov ${ }^{\mathrm{e}}$ \\ G.Matthews ${ }^{\mathrm{e}}$ M.L.Mayoral ${ }^{\mathrm{e}}{ }^{\mathrm{K}}$.McCormick ${ }^{\mathrm{a}}$ A.Meigs ${ }^{\mathrm{e}}$ \\ D.Milanesio ${ }^{\mathrm{g}} \mathrm{I}^{\mathrm{M}}$ Monakhov ${ }^{\mathrm{e}}$ R.Neu ${ }^{\mathrm{a}}$ J.-M.Noterdaeme ${ }^{\mathrm{a}}$ \\ Th.Pütterich ${ }^{\mathrm{a}}$ F.Rimini ${ }^{\mathrm{e}}$ G.Van Rooj ${ }^{\mathrm{h}}$ G.Sergienko ${ }^{\mathrm{b}}$ D.Van Eester ${ }^{\mathrm{f}}$ \\ and JET EFDA contributors ** \\ JET-EFDA, Culham Science Centre, Abingdon, OX14 3DB, UK \\ ${ }^{a}$ Max-Planck-Institut für Plasmaphysik, EURATOM Association, Garching, Germany \\ ${ }^{\mathrm{b}}$ Institute of Energy and Climate Research, Association EURATOM-FZJ, Germany \\ ${ }^{\mathrm{C}}$ CEA, IRFM, F-13108 St Paul-lez-Durance, France \\ ${ }^{\mathrm{d}}$ Association EURATOM-IPPLM, Hery 23, 01-497 Warsaw, Poland \\ ${ }^{\mathrm{e}}$ Culham Science Centre, Association EURATOM-CCFE, Abingdon, Oxon, U.K. \\ ${ }^{\mathrm{f}}$ LPP-ERM/KMS, Association Euratom-Belgian State, TEC partners, Brussels, Belgium \\ ${ }^{\mathrm{g}}$ Associazione EURATOM-ENEA sulla Fusione, Politecnico di Torino, Italy \\ ${ }^{\mathrm{h}}$ Institute for Plasma Physics Rijnhuizen, Association EURATOM-FOM, Nieuwegein, Netherlands
}

\begin{abstract}
.
A variety of plasma wall interactions (PWIs) during operation of the so-called A2 ICRF antennas is observed in JET with the ITER-like wall. Amongst effects of the PWIs, the $W$ content increase is the most significant, especially at low plasma densities. No increase of $W$ source from the main divertor and entrance of the outer divertor during ICRF compared to NBI phases was found by means of spectroscopic and $W I(400.9 \mathrm{~nm})$ imaging diagnostics. In contrary, the $W$ flux there is higher during NBI. Charge exchange neutrals could be excluded as considerable contributors to the $W$ source. The high $W$ content in ICRF heated limiter discharges suggests the possibility of other $W$ sources than the divertor alone. Dependencies of PWIs to individual ICRF antennas during $q_{95}$-scans, and intensification of those for the $-90^{\circ}$ phasing, indicate a link between the PWIs and the antenna near-fields. The PWIs include heat loads and $B e$ sputtering pattern on antenna limiters. Indications of some PWIs at the outer divertor entrance are observed which do not result in higher $W$ flux compared to the NBI phases, but are characterized by small antenna-specific (up to $25 \%$ with respect to ohmic phases) bipolar variations of $W I$ emission. The first TOPICA calculations show a particularity of the A2 antennas compared to the ITER antenna, due to the presence of long antenna limiters in the RF image current loop and thus high near-fields across the most part of the JET outer wall.
\end{abstract}

\footnotetext{
* Corresponding author address: MPI für Plasmaphysik, Boltzmannstr.2, 85748 Garching, Germany; E-mail: bobkov@ipp.mpg.de

** See the Appendix of F. Romanelli et al., Proceedings of the 23rd IAEA Fusion Energy Conference 2010, Daejeon, Korea.
} 
PACS: 52.40.Db, 52.40.Fd, 52.40.Hf, 52.50.Qt

PSI-20 keywords: ICRF, JET, ILW, Sputtering, Power deposition

\section{Introduction}

Heating and current drive system using waves in the Ion Cyclotron Range of Frequencies (ICRF) is an attractive candidate for future fusion devices, because of, among other reasons, unique properties such as selectivity of the heated plasma species and of their energy distribution functions. However, ICRF specific plasma wall interactions (PWIs) which affect the lifetime of plasma-facing components (PFCs) and lead to release of impurities including high$\mathrm{Z}$ elements, need to be controlled. The JET tokamak with its newly operated ITER-like wall (ILW) provides the possibility to study these PWIs with PFC configuration similar to ITER. The whole divertor and the divertor entrance as well as some recessed PFCs on the inner and the outer walls are covered by bulk tungsten $(W)$ or $W$-coated tiles, whereas the first limiting surfaces at the outer and the inner walls are pure beryllium $(B e)$ tiles.

It is known that ICRF operation can lead to deposition of additional heat loads due to RF sheath effects, as has been observed with carbon wall in JET [1]. ICRF operation can also lead to release of $B e$ and high-Z impurities such as $W[2]$ and nickel $N i$. The latter was measurable, but not critical in the carbon JET [3]. This paper presents the first experimental results on characterization of these issues using the so-called A2 ICRF antennas in JET with the ILW. The emphasis is made on impurity release during ICRF, in particular $W$. Some findings from the dedicated papers on ICRF specific Be sputtering [4] and heat loads [5], both being not critical for JET operation, are discussed. A first assessment of the near-fields of the A2 antenna, a likely player in the interactions between the antennas and the wall, is made at the end of the paper. A dedicated comparison of the ICRF performance in JET prior to and with the ILW is made elsewhere [6,7].

On one hand, the change of impurity content in plasma during ICRF can be attributed to an increase of impurity sources at the walls, as in ASDEX Upgrade with $W$-coated ICRF antenna limiters [2]. On the other hand, the impurity content is affected by changes of transport plasma 
properties in the core, due to the heating and effects on the fast particle population [8], or in the scrape-off layer (SOL) by enhanced density convection driven mainly by the RF sheaths [9]. Impurity sources can be driven by 3 main mechanisms: a) sputtering by ions; b) thermal (evaporation, melting); c) sputtering by fast particles including charge exchange (CX) neutrals. The sheath effects [9] are the most likely contributors to (a) and (b). In this respect, a special attention should be paid to the magnetic field line connections going from active ICRF antennas to high-Z PFCs, where the RF sheaths are driven by high antenna near fields, in particular parallel electric field $E_{\|}$.

\section{ICRF system and diagnostics}

Presently the JET ICRF system includes four 4-strap A2 antennas (A, B, C, D, see Fig. 1), with straps powered individually within an antenna. In the experiments reported here, dipole $(0 \pi 0 \pi)$ and $-90^{\circ}(0-\pi / 2-\pi-3 \pi / 2)$ phasings were used, correspondingly with symmetric (dominant $k_{\|} \approx 6.6 \mathrm{~m}^{-1}$ ) and asymmetric (countercurrent, dominant $k_{\|} \approx 3.3 \mathrm{~m}^{-1}$ ) spectrum. Antennas A and B are connected via 3dB hybrid splitters and are operated simultaneously. Only half of antennas A and B (A12 and B12) were used for this study. Hydrogen $(H)$ minority in deuterium $D$ heating scheme at $f_{I C R F}=42 \mathrm{MHz}$ was utilized with a typical $H$ concentration between 2 and $6 \%$.

The concentration of $W$ in the plasma $c_{W Q C}$ is estimated from quasicontinuum (QC) emission at wavelengths around $5 \mathrm{~nm}$ [10], measured by a VUV spectrometer on a vertical line of sight. The emission corresponds to $W$ ionization stages $W 27+$ to $W 35+$. These stages have maximum abundance at $T_{e} \approx 1.5 \mathrm{keV}$. Interpretation of the line radiation of $W 42+$ to $W 45+$ near $6 \mathrm{~nm}$ gives $c_{W L}$ which accounts for a more central $\mathrm{W}$ content at $T_{e}=2$ to $3 \mathrm{keV}$.

Figure 1 presents $W$ source diagnostics. The visible divertor spectroscopy system [11] is used to characterize $W I(400.9 \mathrm{~nm})$ emission and the $W$ source from the main divertor area. A camera system [12] with a narrow-band (1 nm broad) filter and an intensifier is used to characterize the $W I$ emission on a larger area of the outer divertor and its entrance including the top of tile 8 and tile $B$ (see Fig. 1), at which magnetic field connections exist to active ICRF antennas. The 
camera is installed at the outer wall in octant 1 close to the equatorial plane of the torus and provides a view of the divertor and the divertor entrance mostly at octant 2 . The view includes single tiles 8 and tiles $B$ near antenna $\mathrm{A}$ and those near antenna $\mathrm{B}$. The locations are connected to all ICRF antennas along magnetic field lines, due to the large size of the A2 antennas and their limiters. To produce a profile across the outer divertor from the camera images, an array of diagonally arranged 50 spots $(10 \times 10$ pixels each $)$ is used which covers tiles 8 and tiles $B$. The data has to be treated with care, because of the emission near the $W I(400.9 \mathrm{~nm})$ spectral line includes background plasma radiation, mainly due to bremsstrahlung, and reflections from the metallic environment.

A spectroscopy system usually devoted to charge exchange measurements was used to characterize the $B e$ sputtering with additional information from visible video cameras [4]. The JET infrared (IR) camera system [13] includes the cameras looking at antennas A and B which were cross-calibrated against thermocouples and used for heat load studies [5].

\section{Experiments}

The well-developed set of the JET diagnostics provides a good basis for the primary characterization of the ICRF specific PWIs with the ILW.

\subsection{Impurity content}

Fig. 2 shows that application of ICRF power results in radiated power which is higher compared with neutral beam injection (NBI) heating. This comparison is made at the same line average density. In L-modes with ICRF heating close to central deposition, values of $c_{W Q C}$ and $c_{W L}$ indicate that the $c_{W}$ profile is hollow in the center. This is not the case with NBI for which the profile is peaked at low central density with low values of $c_{W}$ at the edge. Cases with off-axis ICRF heating indicate a $c_{W}$ profile close to flat.

From Fig. 2 it can be seen that $W$ is the main radiator during ICRF. Calculation of the power radiated by $W$ is based on flat $c_{W}=c_{W Q C}$ and is somewhat overestimated compared to the 
measured core radiation, because $c_{W L}$ is lower than $c_{W Q C}$. For the flat $c_{W}$ profiles during offaxis heating one estimates $W$ radiation fraction in the range $60 \%$ to $80 \%$. A significant fraction of the rest of the radiated power can be related to nickel $(\mathrm{Ni})$ which behaves similarly to the previous campaigns at JET with carbon wall [3]. Thus both with carbon wall and with the ILW, increase of the $N i$ concentration in JET during ICRF can be considered as having little effect on JET operation.

Interestingly, with the ILW, the high radiation during ICRF operation does not compromise the energy stored in plasma in L-modes. However in the range of densities below the minimum in the L-H threshold power dependence, such as in the case in Fig. 2, the higher radiation during ICRF operation leads to higher power requirement to access H-modes compared to NBI [14].

Fig. 3 presents the dependence of the increase of $c_{W Q C}$ on the line averaged edge density for different ICRF powers and confinement conditions. Indeed, both the density and the ICRF power are the most relevant empirical parameters which influence the $W$ content in the plasma during ICRF. In low density discharges with a toroidal magnetic field of $B_{t}=2.55 \mathrm{~T}$, the concentration is measured at the normalized plasma radius of $\rho=0.5 \pm 0.1$. The radius decreases to $0.3 \pm 0.05$ for higher density and when the magnetic field is decreased to $B_{t}=2.4 \mathrm{~T}$. Although this decrease of the measurement radius can contribute to the decrease of $c_{W Q C}$ when the density is increased, it cannot explain the steep dependence, especially for the $B_{t}=2.4 \mathrm{~T}$ case with off-axis heating. Higher edge density in L-mode means higher $D$ injection rate: in a low triangularity $(\delta=0.27)$ an edge density of $1.7 \cdot 10^{19} \mathrm{~m}^{-3}$ corresponds to a $D$ injection rate of about $2 \cdot 10^{21} \mathrm{el} / \mathrm{s}$. For high power $\left(P_{N B I}>14 \mathrm{MW}, P_{I C R F}=2 M W\right) \mathrm{H}$-mode data from Fig. 3 $c_{W}$ often shows peaked behaviour, so $c_{W Q C}$ represents the $W$ content not completely. For this H-mode data set, data with higher ICRF power are required.

A similar dependence of concentration on edge density can be found for $N i$. Such significant decrease of high-Z concentrations has also been observed with carbon wall in JET [3] and is in line with a number of processes which happen during density increase: a) decrease of impurity source at higher $D$ density, in part due to decrease of concentrations of light impurities; b) change in the SOL density convection; c) change in central plasma transport properties towards worse impurity confinement; d) direct dilution of impurities in the plasma. 


\subsection{W sources}

The relation of central and edge values of $c_{W}$ from Fig. 2 for the ICRF and the NBI phases implies a larger number of $W$ ions crossing the separatrix into the plasma core in the ICRF case. One of the explanations to this could be an increased $W$ source at PFCs, in particular from the divertor, because it has the largest $W$ area interacting with the plasma. However, on the contrary, Fig. 4 for \#81852 demonstrates that deep in the divertor, emission at the $W I$ spectral line which represents the $W$ source at the surfaces, prevails during the NBI phase over that during the ICRF phase. The same also applies to the outer divertor entrance including the top of tile 8 and tile $B$. Both for Fig. 4a (WI spectroscopy) and Fig. 4b (WI imaging), the $W I$ emission is averaged in time during the constant heating power of $3.5 \mathrm{MW}$ during the NBI and the ICRF phases. Such picture is observed for all the NBI vs. ICRF comparisons conducted so far at low to medium densities, where the $W I$ imaging measurements can be applied. A rough estimate using the $W I$ spectroscopy system for cross-calibration to estimate a $W$ flux from the $W I$ emission (assuming constant $S / X B=20$ for simplicity, because uncertainties of the $W I$ imaging and of the $T e$ measurements at the divertor entrance are large), gives a maximum $W$ flux observed by the WI imaging on the top of tile 8 in $\# 81852$ of the order of $3 \cdot 10^{17}$ $\mathrm{m}^{-2} \cdot \mathrm{s}^{-1}$ during the NBI phase. Compared to the value of $5 \cdot 10^{18} \mathrm{~m}^{-2} \cdot \mathrm{s}^{-1}$ averaged over the strike point region, the $W$ influx from the tiles is considerable, because of a higher particle penetration probability to the core plasma from tile 8 and tile $B$ compared to that from the main divertor [16].

The measured $W I$ intensity in the main divertor can be explained solely by the $W$ sputtering by light impurity ions, in particular by $B e$ ions [15]. Measurements from the JET IR camera system do not indicate any ICRF specific thermal release of $W$. It is worth to note that lower surface temperatures in the divertor are measured during the ICRF phases compared to the NBI phases, supposedly due to higher radiated power during ICRF.

The somewhat lower $W$ source during ICRF compared to the NBI phase in \#81852 can be attributed to a lower time-averaged electron temperature $T_{e}$ in the divertor during ICRF at the same heating power and thus lower $W$ sputtering [15]. Due to larger sawteeth during ICRF, variations of $T_{e}$ in the divertor are larger. Selecting the phases during ICRF with the same $T_{e}$ as 
with NBI, the $W$ source at strike points during ICRF is about $25 \%$ higher than that during NBI. One of the candidates to explain this is an increased $B e$ content in the plasma observed during ICRF. Using the $W$ sputtering data by $B e$ [17] with the extended Bohdansky's formula [18] this implies a similar relative increase of $B e$ concentration for $T_{e}$ around $50 \mathrm{eV}$ measured in \#81852 near strike points. Here, spectrosopic measurements show an increase of the BeII line intensity during the ICRF phase by a factor compared to the NBI phase. Measurements of $Z_{e f f}$ indicate about a factor of two higher $B e$ level in the central plasma in the ICRF phase as well. This relative increase of $B e$ level is more than required to explain the $25 \%$ higher $W$ sputtering at fixed $T_{e}$, assuming that the high ionization stages of $B e$ follow the same trend. This adds to the other indications [15] that changes of the measured $B e$ level are not fully consistent with changes of the $W$ sputtering yield, implying that other parameters may also be important.

Although no additional $W$ source is measured during ICRF phases, there are some indications of ICRF specific interactions at the entrance of the outer divertor. This can be seen in the series of discharges with $q_{95}$-scan and modulated ICRF pulses of $1 \mathrm{MW}$ from individual antennas. The $W I$ imaging measurements on the top of tile 8 near antenna $B$ (see Fig. 1) are summarized in Fig. 5 in terms of relative changes of $W I$ emission intensity during ICRF with respect to the ohmic phases $\Delta I_{R F} / I_{\text {ohmic }}$ at the same $q_{95}$-value. The maximum observed value $\Delta I_{R F} / I_{\text {ohmic }}=0.25$ corresponds to a change of $W$ flux by $\approx 10^{17} \mathrm{~m}^{-2} \cdot \mathrm{s}^{-1}$. Fig. 5 indicates more complex interactions than just an increase of the $W$ source, especially in the case of the $-90^{\circ}$ phasing (Fig. 5b) where the WI emission decreases at higher $q_{95}$ values. Individual responses to different antennas can be seen, less in the dipole case with the high $\delta$ plasma shape $(\delta=0.42)$ in Fig. $5 \mathrm{c}$ for which the magnetic field lines starting at the antennas divert towards the deeper regions of the divertor. The difference between the antennas are distinctive in Fig. 5a,b. However, the shapes of the WI emission dependencies on $q_{95}$ have some degree of similarity between each other, making it difficult to distinguish a possible spatial $W$ sputtering pattern from consequences of a more global change of the SOL during the $q_{95}$-scan. These WI imaging measurements are well reproducible from discharge to discharge. The issue of the background plasma emission is likely not to play a significant role in these cases, because utilization of an additional background subtraction, based on the intensity in the gap between tile 8 and tile $B$ and the assumption on local behaviour of the $W I$ emission near the PFCs, leads to similar 
results, albeit with larger error bars. The influence of reflections can not be fully excluded.

Thus, although there is an increase of $W$ content when ICRF is switched on compared to pure NBI phases, the measurements of $W I$ emission from the main divertor area and from the entrance of the outer divertor do not corroborate the picture of an increased $W$ source from the divertor at fixed heating power. A strong toroidal asymmetry of ICRF-specific $W$ sources which would not be covered by $W I$ imaging camera during the $q_{95}$-scan could in theory explain this behaviour. Given the variety of the toroidal locations scanned during the $q_{95}$-scans, this would imply a very strong and very localized $W$ source. Other wall components, not visible by the diagnostics, are potential $W$ sources. These include tile $C$ - a likely contributor, and a number of outer and inner wall $W$-coated components - less likely contributors, because most of them are recessed behind the plasma limiting surfaces. So far no consistent observation of significant changes of $W$ content in the plasma exists for changes of the clearance between the plasma and the outer wall. Interesting observations exist in limiter discharges. Even in limiter plasmas the ICRF phases are characterized with higher $c_{W}$ compared to the NBI phases. The ICRF discharges with $P_{I C R F}=2 \mathrm{MW}$ are represented by filled black circles in Fig. 3. The values of $c_{W Q C}$ are well within the ballpark of the divertor discharges at the same line average edge density. Moreover, when in such a limiter discharge the plasma is shifted upwards away from the divertor, providing better magnetic isolation between the divertor and the antennas, $c_{W Q C}$ does not decrease. If $W$ sputtering by CX neutrals was excluded, this would suggest that $W$ sources above the divertor can play a role.

\subsection{Role of CX neutrals in $W$ sputtering}

In fact, for the energy range of up to $30 \mathrm{keV}$, the fast particle analyzer data favors higher $W$ release by charge exchange particles in the case of NBI heating. In this energy range most of the fast particles are detected and the $W$ sputtering yield is highest [18]. The ICRF phases are characterized by significantly higher neutral $H$ flux compared to NBI, whereas the NBI phase are accompanied by significantly higher $D$ flux compared to ICRF. For \#81852, where the density is low and the detected CX neutral fluxes are high, one estimates with the help of the energy dependence of the $W$ sputtering yield from [18], that $8.7 \cdot 10^{11} \mathrm{~m}^{-2} \cdot \mathrm{s}^{-1} W$ flux 
(integrated over the energy range) is due to neutral $H$ in the ICRF case, and $9 \cdot 10^{12} \mathrm{~m}^{-2} \cdot \mathrm{s}^{-1}$ of $W$ flux is due to neutral $D$ in the NBI case. Even considering a large potentially affected area, these numbers are too low to play a significant role for the $W$ content in the plasma, assuming an isotropic distribution of the particles in the relevant energy range up to $30 \mathrm{keV}$. However, based on the data available, no statement can be made about fluxes of $\mathrm{CX} B e$ particles and their effect on $W$ sputtering and thus CX cannot be fully excluded.

\subsection{Role of sheath effects}

A variety of observations has been made in the experiments which depend on antenna phasing. The $-90^{\circ}$ phasing of the ICRF antennas is generally characterized by intensified PWIs compared to the dipole case. Fig. 6 shows this for the $W$ content per MW of ICRF power. Other observations during the $-90^{\circ}$ phasing include increased heat loads at the antenna PFCs [5] and increased $B e$ sputtering at the antenna limiters [4].

The maximum heat load at the antenna PFCs increases about linearly with the product of the near-antenna density and the ICRF power. This implies a modification of density in front of the antennas by ICRF power [5] which could be attributed to convective cells $[19,20]$. The spectroscopic observations of the increased $B e$ sputtering at the limiters are consistent with the spatial sputtering patterns moving in accordance with magnetic field connections to active ICRF antennas. Thus the observations of the local heat loads and the $B e$ sputtering patterns hint directly to the enhanced sheath phenomena.

Apart from the phasing dependence from Fig. 6, the limited experimental data obtained so far does not allow to pin down the sheath effects as responsible for the increased $W$ content. Nevertheless, at least for the studies of the Be sputtering and the heat loads [4,5], it is important to assess the near fields of the A2 antenna which drive the RF sheaths. This assessment should also prove useful for the $W$ studies in the future. A similar assessment for the ITER antenna [22] is taken as a reference.

Fig. 7 shows first $E_{\|}$calculations for the A2 antenna using TOPICA [23] (same code as used to model the ICRF antennas in ITER) with a 3D antenna model adapted to flat geometry and 
JET L-mode plasma density and temperature profiles. Scaled to ITER relevant ICRF power, $E_{\|}$absolute values for the A2 antenna are a factor of $>2$ higher than the $E_{\|}$values in front of the ITER antenna for the high density reference profiles [22]. A distinctive feature of $E_{\|}$ at the A2 antenna is the distribution of the fields along the whole length of the long poloidal limiters at JET. The long poloidal limiters are substantial parts of the A2 antenna image RF current loop in JET. This is because of limited other surfaces available for the RF currents to flow, strengthened by the fact that the antenna PFCs are connected to the antenna boxes via narrow shorts close to the antenna-plasma interface. Thus $E_{\|}$cover almost the whole height of the outer wall at JET starting down at the divertor entrance. Design of such protruding antenna has to be optimized. The design strategies include: a) increased RF conducting surfaces to short circuit RF image currents; b) antenna strap arrangement to better compensate the $(0 \pi)$ phased image current contributions. An example of such approach is the planned 3-strap antenna in ASDEX Upgrade [21].

The calculations indicate that the experience from JET should be taken with a double care when discussed in the context of the ITER ICRF system, because the distinctive features of the near fields of the A2 antenna are not representative of the ITER antenna design. In ITER, the ICRF antenna will not be protruding, which should give much lower area affected by $E_{\|}$than for the A2 antenna, both on absolute and on relative spatial scale.

The TOPICA near-field calculations alone give only the first idea on how the antennas can behave in terms of sheath effects. To compare calculations with experiments, advanced approaches for non-linear sheath modelling such as in $[22,24]$ have to be further developed.

\section{Summary}

First experimental observations in JET with the ILW on the interactions between the ICRF antennas and the ILW have been obtained. For JET operation, increased $B e$ sputtering and increased local heat loads at the antenna PFCs during ICRF do not have any impact, whereas an increased $W$ content has an influence at low densities, when the $W$ content is highest.

However the reasons for the increased $W$-content remain uncertain. No increased $W$ source 
during ICRF compared to NBI was found in the main divertor area and at the outer divertor entrance from the $W I(400.9 \mathrm{~nm})$ emission measurements. Observations of the $W I$ emission at the outer divertor entrance show individual bipolar responses to the power from particular ICRF antennas, with more variations during the $90^{\circ}$ phased operation. A high $W$ content in the limiter plasmas hints at other possible $W$ sources than the observable divertor area. The charge-exchange hydrogen and deuterium neutrals could be excluded as strong contributors to the $W$ source, assuming their isotropic distribution for the energies below $30 \mathrm{keV}$. The same could not be done so far for $B e$ neutrals. Phasing dependence of observations on $W, B e$ and the heat loads, with evidences of importance of magnetic field line connection to active antennas, points on the RF near field phenomena. However, neither the effect on $W$ source, nor the effect of ICRF induced SOL changes on $W$ penetration inside the core plasma could be confirmed or excluded.

First TOPICA runs with an adapted A2 antenna model shows distribution of parallel RF electric field alongside the whole height of the antenna limiters which cover most of the SOL starting from the outer divertor entrance. This is different to the ITER antenna design where the near fields will be better contained within the antenna vicinity. Thus the JET ICRF experimental experience with the ILW can not be directly applied to the ITER ICRF antenna without further studies. The studies will require more experimental data from JET with better diagnostic coverage as well as advanced sheath modeling.

\section{Acknowledgements}

This work was supported by EURATOM and carried out within the framework of the European Fusion Development Agreement. The views and opinions expressed herein do not necessarily reflect those of the European Commission.

\section{References}

[1] P. Jacquet et al., Nucl. Fusion 51 (2011) 103018. 
[2] V1. Bobkov et al., Journal of Nuclear Materials 415 (2011) S1005.

[3] A. Czarnecka et al, accepted to Plasma Phys. Control. Fusion, IOP preprint EFDA-JET-PR(11)17.

[4] C. Klepper, this conference, Poster P1-86.

[5] Ph. Jacquet, this conference, Poster P1-20.

[6] D. Van Eester et al., Europhysics Conference Abstracts, (Proc. of the 39rd EPS Conference on Plasma Physics, Stockholm, Sweden, 2012) 36F (2012) P.1.094.

[7] M.L. Mayoral et al., Proc. of the 24th IAEA Fusion Energy Conference, San Diego, 2012, EX/4-3.

[8] C. Angioni, Phys. Plasmas textbf14 (2007) 055905.

[9] D.A. D’Ippolito and J.R. Myra, Journal of Nuclear Materials 415 (2011) S1001.

[10] T. Pütterich et al., Plasma Phys. Control. Fusion 50 (2008) 085016.

[11] A. Meigs, this conference, Poster P1-89.

[12] A. Huber et al, submitted to Rev. Sci. Instrum (HTPD conference) (2012).

[13] G. Arnoux et al., Proc. of 27th Symposium on Fusion Technology (SOFT), Liege, Belgium, 2012.

[14] C. Maggi et al., EPS Conference 2012.

[15] G. Van Rooj et al., this conference, Invited talk I-3.

[16] J. Strachan et al., W screening factors, private communication.

[17] C. Bjorkas et al., Calculated parameters for sputtering yield of $W$ by $B e$, private communication.

[18] R. Behrisch, W. Eckstein (Eds.), Sputtering yields, Springer, Springer-Verlag (2007).

[19] L. Colas et al., Nucl. Fusion 45 (2005) 767.

[20] I. Cziegler et al., Plasma Phys. Control. Fusion 54 (2012) 105019.

[21] A. Kallenbach et al., IEEE Transactions on Plasma Science 40 (2012) 605.

[22] J. Jacquot et al., AIP conf. Proc. 1406 (19th RF top. conf. 2011) 211.

[23] V. Lancellotti et al., Nucl.Fusion 46 (2006) S476.

[24] D.A. D'Ippolito et al., AIP conf. Proc. 1406 (19th RF top. conf. 2011) 195. 
Fig. 1. Arrangement of ICRF antennas and $W$ source diagnostics in the divertor.

Fig. 2. Comparison of ICRF and NBI heating in \#81852 with $B_{T}=2.55 \mathrm{~T}$ and $f_{I C R F}=$ $42 \mathrm{MHz}$. Upper graph: solid lines without symbols - applied ICRF and NBI power, crosses - radiated power estimated using $c_{W Q C}$, dashed line - measured core radiation. Lower graph: solid line with crosses $-c_{W Q C}$ measured at $\rho_{Q C}=0.6 \pm 0.1$ during ICRF and at $\rho_{Q C}=0.5 \pm 0.2$ during NBI; solid line with triangles $-c_{W L}$ measured at $\rho_{L}=0.25 \pm 0.1$ during ICRF and at $\rho_{L}=0.2 \pm 0.1$ during NBI.

Fig. 3. Dependence of change of $c_{W Q C}$ during ICRF on line averaged edge density. Open squares, diamonds and triangles: $B_{T}=2.55 \mathrm{~T}, I_{p}=2.5 \mathrm{MA}$, low $\delta$ shape; stars, filled squares, diamonds and triangles: $B_{T}=2.4 \mathrm{~T}, I_{p}=2 \mathrm{MA}$, low $\delta$ shape; open circles: $B_{T}=2.7 \mathrm{~T}, I_{p}=2.5 \mathrm{MA}$, high $\delta$ shape; closed circles: $B_{T}=2.5 \mathrm{~T}, I_{p}=2 \mathrm{MA}$, limiter shape.

Fig. 4. WI emission at $400.8 \mathrm{~nm}$ from the $W I$ spectroscopy (a) and the $W I$ imaging (b) in \#81852 for ohmic only, ICRF and NBI heated phases (3.5 MW phases). Top of tile 8 is indicated, which appears larger on the WI imaging system due to viewing geometry.

Fig. 5. Relative change of WI intensity at the top of tile 8 near antenna B during $1 \mathrm{MW}$ of ICRF with respect to ohmic phases, deduced from the $W I$ imaging: a) low $\delta$ with dipole; b) low $\delta$ with $-90^{\circ}$; c) high $\delta$ with dipole.

Fig. 6. Comparison of $c_{W Q C}$ in the dipole and the $-90^{\circ} \mathrm{ICRF}$ (only A and B) powered L-modes.

Fig. 7. TOPICA calculations of $E_{\|}$in front of $(0 \pi 0 \pi)$ phased JET A2 antenna in L-mode, normalized to $1 \mathrm{MW}$ coupled to antenna. 


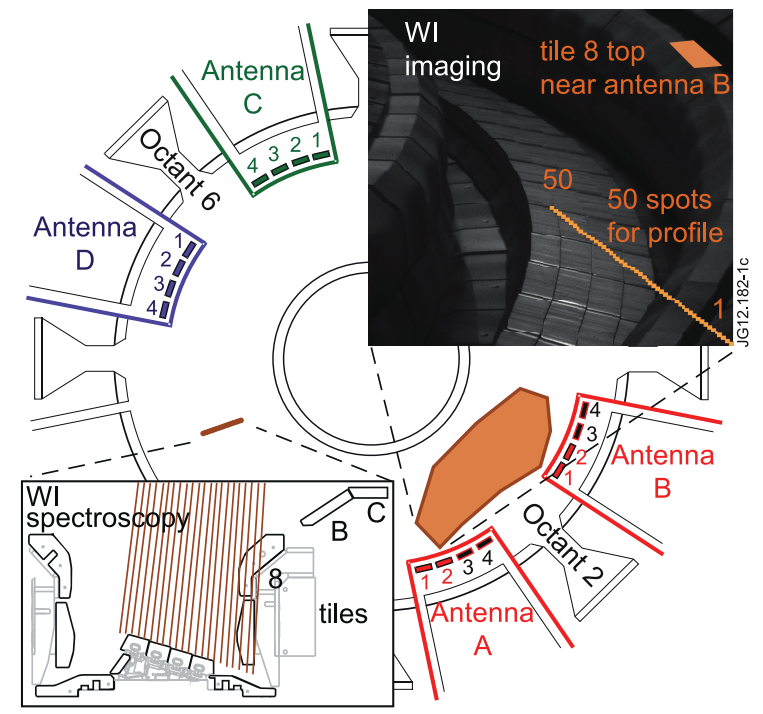

Fig. 1. 


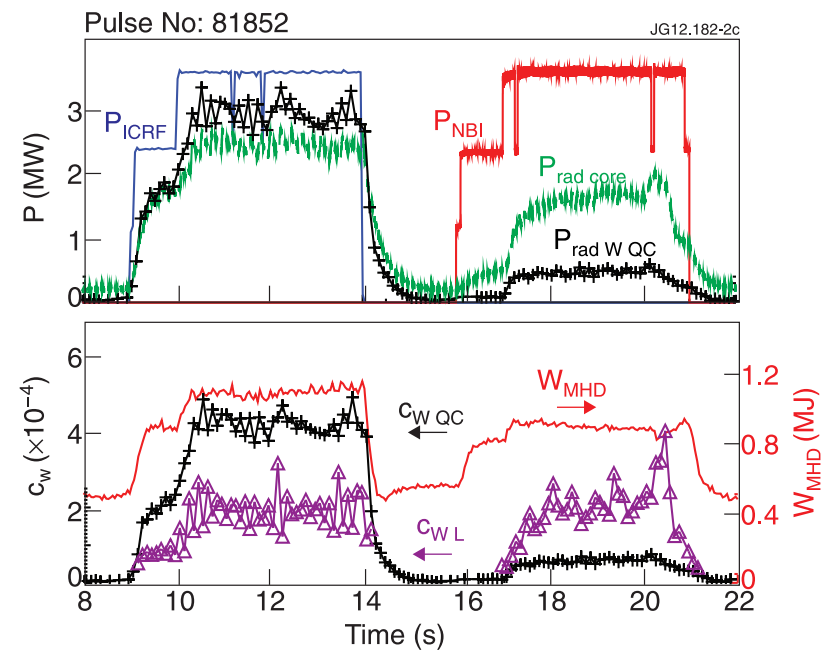

Fig. 2. 


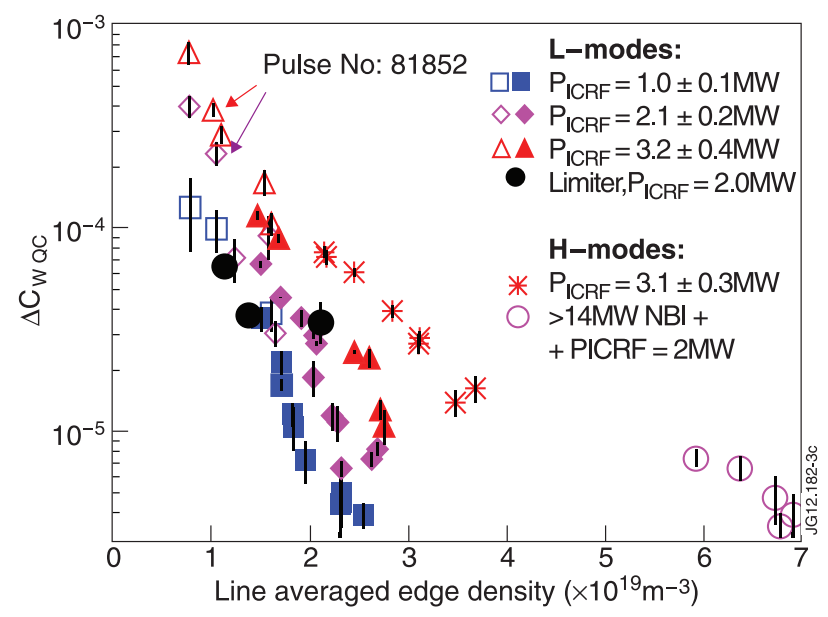

Fig. 3. 

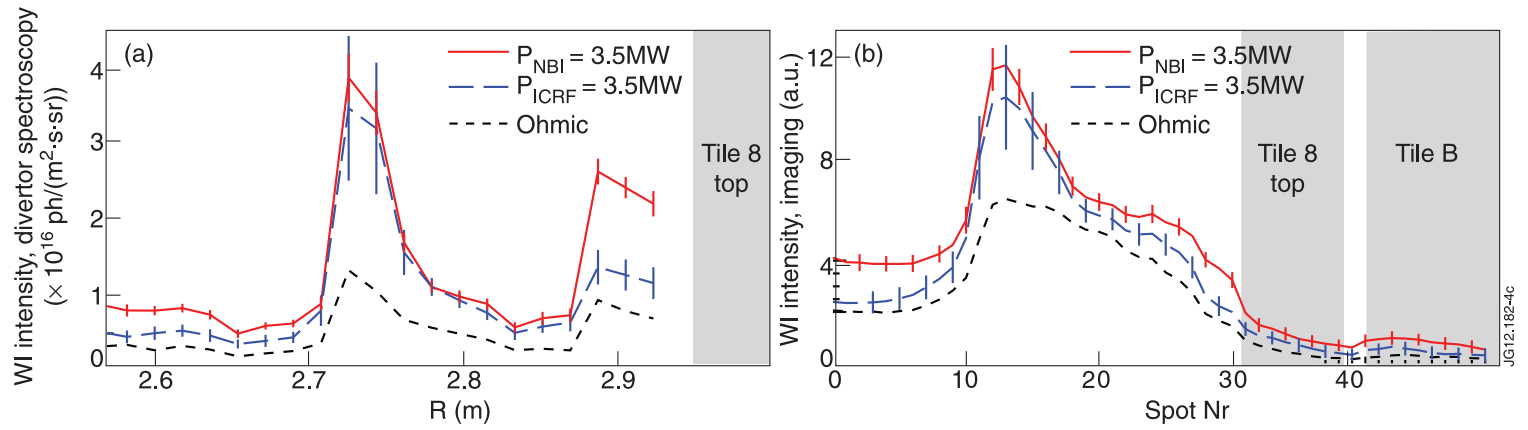

Fig. 4. 


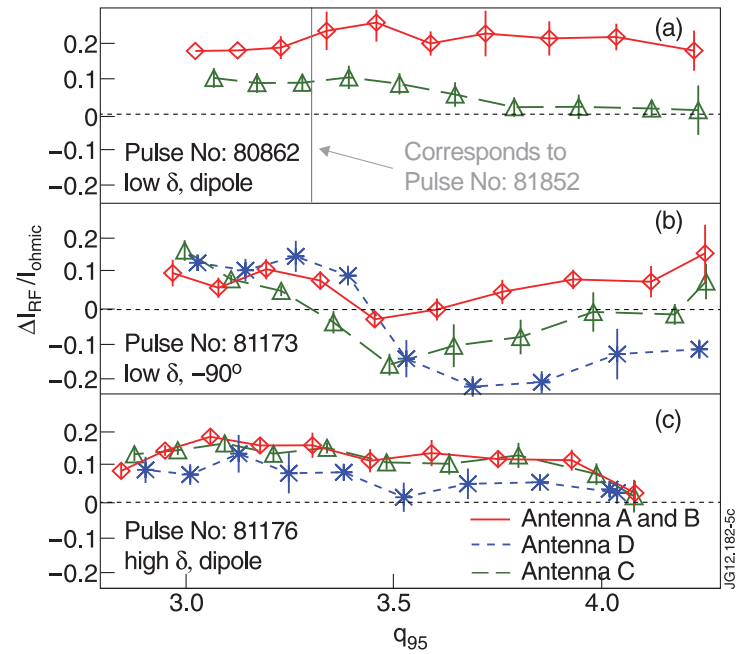

Fig. 5. 


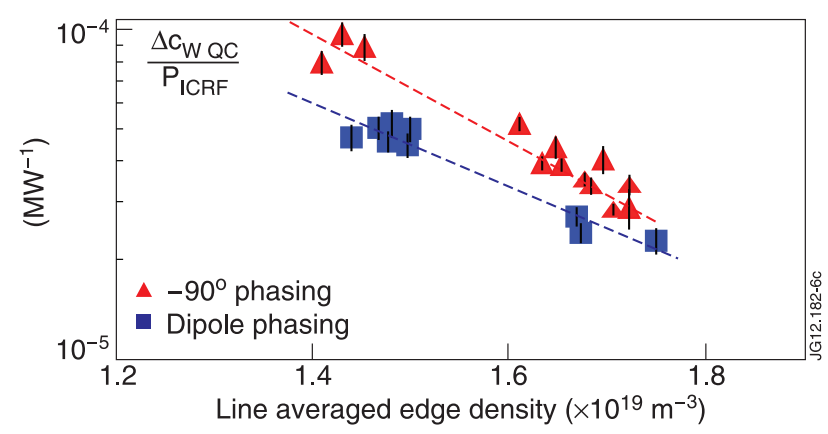

Fig. 6. 


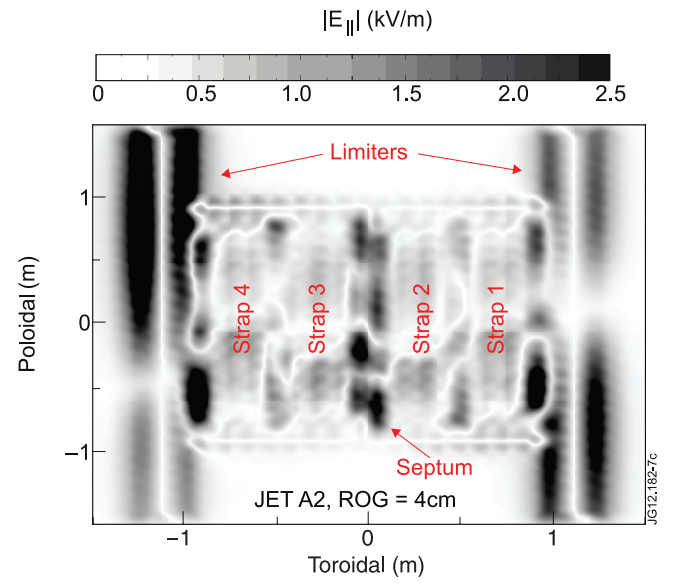

Fig. 7. 